\title{
Massive Fields with Arbitrary Integer Spin in Symmetrical Einstein Space
}

\author{
S. M. Klishevich* \\ Institute for High Energy Physics \\ Protvino, Moscow Region, 142284, Russia
}

June 28, 2021

\begin{abstract}
We study the propagation of gauge fields with arbitrary integer spins in the symmetrical Einstein space of any dimensionality. We reduce the problem of obtaining a gauge-invariant Lagrangian of integer spin fields in such background to algebraic problem of search for a set of operators with certain features by means of the representation of higher-spin fields in the form of vectors in pseudo-Hilbert space. We consider such construction at linear order in the Riemann tensor and scalar curvature and also present an explicit form of interaction Lagrangians and gauge transformations for massive particles of spins 1 and 2 in terms of symmetrical tensor fields.
\end{abstract}

PACS number(s): 11.10.Kk, 11.10.Lm, 11.15.-q, 11.15.Ex

Keywords: Massive higher spin fields; Gauge interactions

${ }^{*}$ E-mail address: klishevich@mx.ihep.su 


\section{Introduction}

Problems of obtaining a consistent description of the gravitational interactions of higher-spin fields have the particular significance since it allows one to connect the higher-spin fields with the observable world.

It is well known that the gravitational interaction of massless fields with spins $s \geq 2$ does not exist in an asymptotically flat space-time [1]. For the covariant description of physical fields one must replace the ordinary derivatives in the Lagrangian and gauge transformations with the covariant ones. Since the covariant derivatives do not commute, the gauge invariance fails and a residual appears. For the fields with spins $s \geq 2$ the residual is proportional to the Riemann tensor. In general, one cannot cancel such a residual in an asymptotically flat space-time in linear approximation by any changes of the Lagrangian and transformations. Therefore, in such case this approximation does not exist. Since linear approximation does not depend on the presence of any other fields in the system, this means that the whole theory of interaction does not exist either.

This problem can be overcome in several ways. For instance, one can consider the massless fields in a space of constant curvature. In this case the Lagrangian for gravity would have the additional term $\delta \mathcal{L} \sim \sqrt{-g} \lambda$, where $\lambda$ is the cosmological constant. A modification of the Lagrangian and the transformations leads to a mixing of terms with different numbers of the derivatives. This allows one to compensate the residual with terms proportional to $R_{\mu \nu \alpha \beta}$. The complete theory will be represented as series in inverse value of the cosmological constant [2, [2]. This means the non-analyticity of the theory in $\lambda$ at zero, i.e. the impossibility of a

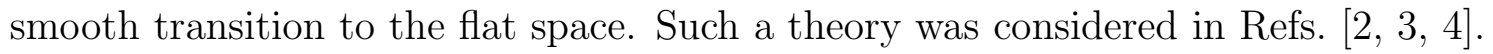

Besides, massive higher-spin fields can have the gravitational interaction. For example, the string theory represents the consistent gravitational interaction of the massive higher-spin fields. In Ref. [5] the interaction of the fields at linear order was derived while investigating three point functions ot the type II superstring, which contains one graviton and two massive states.

In the literature the gravitational interaction of arbitrary spin fields were considered at the lowest order in the Riemann tensor [6, 7, 5]. Considering the interactions, the authors started from the free theory of the massive fields in the conventional form [8]. The "minimal" introduction of the interaction leads to contradictions, therefore, it is necessary to consider non-minimal terms in the interaction Lagrangian. Since, the conventional Lagrangian [8] for the massive spin- $s$ fields is not gauge invariant, in this approach there are no restrictions on the form of the non-minimal interaction. But, in a general case, such a theory is pathological, therefore, to build a consistent theory of the interaction it is necessary to introduce an additional restrictions on the non-minimal terms. So, for instance, when investigating the gravitational interactions [6, 7], the authors required for the theory to have the tree-level unitarity up to the Planck scale.

In our opinion it is more convenient to use the gauge-invariant approach when one analyzes an interaction of the massive fields [9, 10, 11], [12] or [13]. Under such an approach the interaction is considered as a deformation of initial gauge algebra and Lagrangian' [14, 11]. Although, generally speaking, the gauge invariance does not ensure the consistency of mas-

\footnotetext{
${ }^{1}$ Of course, one must consider only a non-trivial deformation of the free algebra and Lagrangian which cannot be completely gauged away or removed by a redefinition of the fields.
} 
sive theories, but, anyway, it allows one to narrow the searches and conserves the appropriate number of physical degree of freedom. Besides, such an approach is quite convenient and practical.

Here we go along the line of Refs. [15, 16] where the electromagnetic interaction of the massive fields with integer and half-integer spins was investigated. We represent a free state with the arbitrary integer spin $s$ as state $\left|\Phi^{s}\right\rangle$ in a Pseudo-Hilbert space [15]. The tensor fields corresponding to the particle with spin $s$ are coefficient functions of the state $\left|\Phi^{s}\right\rangle$. On the considered Fock space we introduce a set of operators by means of which we define the gauge transformations and necessary constraints for the state $\left|\Phi^{s}\right\rangle$. The gauge-invariant Lagrangian has the form of the expectation value of the Hermitian operator, which consists of the operators, in the state $\left|\Phi^{s}\right\rangle$.

In the considered approach the gauge invariance is a consequence of commutation relations of the introduced operators. For the covariant description of fields in the Riemann background, one must replace the ordinary derivatives with the covariant ones. This leads to a change of algebraic features of the operators and, as a consequence, to the loss of the gauge invariance for the higher-spin fields. We reduce the problem of recovering the invariance to algebraic problem of search for such modified operators that depend on the Riemann tensor and scalar curvature and satisfy the same commutation relations as initial operators in the flat space. In this, we should note that in the massless case one cannot realize such a construction in an asymptotically flat space. In section 3, for the massive theory we construct the set of the operators having the algebraic features of the free ones at linear order in the Riemann tensor and scalar curvature. Besides, in the next section we give an explicit form of interaction Lagrangian and transformations for the massive vector and spin-2 fields.

\section{$1 \quad$ Free Field with Spin $s$}

Massless fields. Let us consider the Fock space generated by the creation and annihilation operators $\bar{a}_{\mu}$ and $a_{\mu}$, which are vectors on the $D$-dimensional Minkowski space $\mathcal{M}_{D}$ and which satisfy the following algebra

$$
\left[a_{\mu}, \bar{a}_{\nu}\right]=g_{\mu \nu}, \quad a_{\mu}^{\dagger}=\bar{a}_{\mu},
$$

where $g_{\mu \nu}$ is the metric tensor with the signature $\left\|g_{\mu \nu}\right\|=\operatorname{diag}(-1,1,1, \ldots, 1)$. Since the metric is indefinite, the Fock space that realizes the representation of Heisenberg algebra (1) is Pseudo-Hilbert.

Let us consider the state in the introduced space:

$$
\left|\Phi^{s}\right\rangle=\frac{1}{\sqrt{s}} \Phi_{\mu_{1} \ldots \mu_{s}}(x) \prod_{i=1}^{s} \bar{a}_{\mu_{i}}|0\rangle .
$$

Coefficient function $\Phi_{\mu_{1} \ldots \mu_{s}}(x)$ is a symmetrical tensor field of rank $s$ on space $\mathcal{M}_{D}$. For this tensor field to describe the state with spinf $s$ one has to imposes the condition:

$$
\Phi_{\mu \mu \nu \nu \mu_{4} \ldots \mu_{s}}=0 \text {. }
$$

\footnotetext{
${ }^{2}$ The representation of the free fields with arbitrary integer spins in such a form was considered in Refs. 17, 12, 18

${ }^{3}$ We consider symmetric tensor fields only.
} 
In terms of such fields the Lagrangian [19, 20] has the form

$$
\begin{aligned}
\mathcal{L}_{s}= & \frac{1}{2}\left(\partial_{\mu} \Phi^{s}\right) \cdot\left(\partial_{\mu} \Phi^{s}\right)-\frac{s}{2}\left(\partial \cdot \Phi^{s}\right) \cdot\left(\partial \cdot \Phi^{s}\right)-\frac{s(s-1)}{4}\left(\partial_{\mu} \Phi^{\prime s}\right) \cdot\left(\partial_{\mu} \Phi^{\prime s}\right) \\
& -\frac{s(s-1)}{2}\left(\partial \cdot \partial \cdot \Phi^{s}\right) \cdot \Phi^{\prime s}-\frac{1}{8} s(s-1)(s-2)\left(\partial \cdot \Phi^{\prime s}\right) \cdot\left(\partial \cdot \Phi^{\prime s}\right) .
\end{aligned}
$$

The following notation $\Phi^{\prime}=\Phi_{\mu \mu \ldots}$ have been used here while the point means the contraction of all indexes $\Phi^{s} \cdot \Phi^{s}=\Phi_{\mu_{1} \ldots \mu_{s}} \Phi^{\mu_{1} \ldots \mu_{s}}$.

This Lagrangian is invariant under the transformations

$$
\begin{aligned}
\delta \Phi_{\mu_{1} \ldots \mu_{s}} & =\partial_{\left(\mu_{1}\right.} \Lambda_{\left.\mu_{2} \ldots \mu_{s-1}\right)} \\
\Lambda_{\mu \mu \mu_{3} \ldots \mu_{s-1}} & =0
\end{aligned}
$$

Let us introduce the following operators on our pseudo-Hilbert space

$$
L_{1}=p \cdot a, \quad L_{-1}=L_{1}^{\dagger}, \quad L_{2}=\frac{1}{2} a \cdot a, \quad L_{-2}=L_{2}^{\dagger}, \quad L_{0}=p^{2} .
$$

Here $p_{\mu}=i \partial_{\mu}$ is the momentum operator that acts on the space of the coefficient functions.

Operators of type (7) appear as constraints of a two-particle system under quantizationf [21]. This operators satisfy the commutation relations:

$$
\begin{aligned}
& {\left[L_{1}, L_{-2}\right]=L_{-1}, \quad\left[L_{1}, L_{2}\right]=0 \text {, }} \\
& {\left[L_{2}, L_{-2}\right]=N+\frac{D}{2}, \quad\left[L_{0}, L_{n}\right]=0} \\
& {\left[L_{1}, L_{-1}\right]=L_{0}, \quad\left[N, L_{n}\right]=-n L_{n}, \quad n=0, \pm 1, \pm 2 \text {. }}
\end{aligned}
$$

Here $N=\bar{a} \cdot a$ is the level operator that defines the spin of states. So, for instance, for state (2)

$$
N\left|\Phi^{s}\right\rangle=s\left|\Phi^{s}\right\rangle
$$

In terms of operators (8) condition (3) can be written as

$$
\left(L_{2}\right)^{2}\left|\Phi^{s}\right\rangle=0
$$

while gauge transformations (5) take the form

$$
\delta\left|\Phi^{s}\right\rangle=L_{-1}\left|\Lambda^{s-1}\right\rangle
$$

Here, the gauge state

$$
\left|\Lambda^{s-1}\right\rangle=\Lambda_{\mu_{1} \ldots \mu_{s-1}} \prod_{i=1}^{s-1} \bar{a}_{\mu_{i}}|0\rangle
$$

satisfies the condition

$$
L_{2}|\Lambda\rangle=0
$$

This condition is equivalent to (6) for the coefficient functions.

\footnotetext{
${ }^{4}$ It is also possible to regard operators $(7)$ as a truncation of the Virasoro algebra.
} 
Lagrangian (4) can be written as the expectation value of a Hermitian operator in state (2)

$$
\mathcal{L}_{s}=\left\langle\Phi^{s}|\mathcal{L}(L)| \Phi^{s}\right\rangle, \quad\left\langle\Phi^{s}|=| \Phi^{s}\right\rangle^{\dagger},
$$

where

$$
\begin{aligned}
\mathcal{L}(L)= & L_{0}-L_{-1} L_{1}-2 L_{-2} L_{0} L_{2}-L_{-2} L_{-1} L_{1} L_{2} \\
& +\left\{L_{-2} L_{1} L_{1}+\text { h.c. }\right\} .
\end{aligned}
$$

Lagrangian (12) is invariant under transformations (10) as a consequence of the relation

$$
\mathcal{L}(L) L_{-1} \sim(\ldots) L_{2} .
$$

Massive fields Let us consider the massive states of arbitrary spin $s$ in the similar manner. For that we have to extend our Fock space by introducing scalar creation and annihilation operators $\bar{b}$ and $b$, which satisfy the usual commutation relations

$$
[b, \bar{b}]=1, \quad b^{\dagger}=\bar{b} .
$$

Operators (7) are modified as follows:

$$
L_{1}=p \cdot a+m b, \quad L_{2}=\frac{1}{2}\left(a \cdot a+b^{2}\right), \quad L_{0}=p^{2}+m^{2} .
$$

Here $m$ is an arbitrary parameter having the dimensionality of mass. In the non-interacting case one can consider such transition as the dimensional reduction $\mathcal{M}_{D+1} \rightarrow \mathcal{M}_{D} \otimes S^{1}$ with the radius of compactification $R \sim 1 / m$ (refer also to [12, [18]).

We shall describe the massive field with spin $s$ as the following vector in the extended Fock space:

$$
\left|\Phi^{s}\right\rangle=\sum_{n=0}^{s} \Phi_{\mu_{1} \ldots \mu_{n}}(x) \bar{b}^{s-n} \prod_{i=1}^{n} \bar{a}_{\mu_{i}}|0\rangle .
$$

Like the massless field case, this state satisfies the same condition (9), but in terms of operators (15). The algebra of operators (8) changes insignificantly, the only commutator modified is

$$
\left[L_{2}, L_{-2}\right]=N+\frac{D+1}{2} .
$$

Here, as in the massless case, the operator $N=\bar{a} \cdot a+\bar{b} b$ defines the spin of massive states. The Lagrangian describing the massive field with spin $s$ has the form (13) as well, where the expectation value is taken in state (16). Such Lagrangian is invariant under transformations (10) with the gauge Fock vector

$$
\left|\Lambda^{s-1}\right\rangle=\sum_{n=0}^{s-1} \Lambda_{\mu_{1} \ldots \mu_{n}} \bar{b}^{s-n-1} \prod_{i=1}^{n} \bar{a}_{\mu_{i}}|0\rangle,
$$

which satisfies condition (匼). 


\section{Propagation of Massive higher-spin Field in Sym- mertical Einstein Space}

In this section we consider an arbitrary $D$-dimensional symmetrical Einstein space, i.e. the Riemann space defined by the following equations:

$$
\begin{aligned}
\mathcal{D}_{\mu}^{(\Gamma)} R_{\nu \alpha \beta \gamma} & =0, \\
R_{\mu \nu}-\frac{1}{2} g_{\mu \nu} R & =g_{\mu \nu} \lambda,
\end{aligned}
$$

where $\mathcal{D}_{\mu}^{\Gamma}$ is the covariant derivative with the Cristofel connection $\Gamma^{\alpha}{ }_{\nu \mu}$. We assume that the Greek indexes are global while the Latin ones are local. As usual, the derivative $\mathcal{D}_{\mu}^{\Gamma}$ acts on tensor fields with global indexes only.

To describe the massive higher-spin fields in the Riemann background, we must replace the ordinary derivatives with the covariant one, i.e. we make the substitution:

$$
p_{\mu} \rightarrow \mathcal{P}_{\mu}=i\left(\mathcal{D}_{\mu}^{\Gamma}+\omega_{\mu}^{a b} \bar{a}_{a} a_{b}\right)
$$

where $\omega_{\mu}^{a b}$ is the Lorentz connection. We imply that the creation and annihilation operators primordially carry the local indexes. We also have to introduce the non-degenerate vielbein $e_{\mu}^{a}$ for the transition from the local indexes to the global ones and vice versa. As usual, we impose the conventional requirement on the vielbein

$$
\mathcal{D}_{\mu}^{(\Gamma+\omega)} e_{\nu}^{a}=\partial_{\mu} e_{\nu}^{a}-\Gamma_{\nu \mu}^{\lambda} e_{\lambda}^{a}+\omega_{\mu}^{a} b e_{\nu}^{b}=0 .
$$

By means of this relation one can transfer from expressions with one connection to those with other. Besides, we should note that due to this relation the operator $\mathcal{P}_{\mu}$ commutes with the vector creation-annihilation operators carrying global indexes $\bar{a}_{\nu}=e_{\nu}^{b} \bar{a}_{b}$ and $a_{\nu}=e_{\nu}^{b} a_{b}$. This allows us not to care about the ordering of operators (15).

One can verify that the covariant momentum operator defined in this way properly acts on the states of type (16), indeed

$$
\mathcal{P}_{\mu}|\Phi\rangle=i \mathcal{D}_{\mu}^{(\omega)} \Phi^{b_{1} \ldots b_{n}} \prod_{i=1}^{n} \bar{a}_{b_{i}}|0\rangle=i \mathcal{D}_{\mu}^{(\Gamma)} \Phi^{\nu_{1} \ldots \nu_{n}} \prod_{i=1}^{n} \bar{a}_{\nu_{i}}|0\rangle .
$$

The commutator of the covariant momenta defines the Riemann tensor:

$$
\left[\mathcal{P}_{\mu}, \mathcal{P}_{\nu}\right]=R_{\mu \nu}^{a b}(\omega) \bar{a}_{a} a_{b}
$$

where $R_{\mu \nu}^{a b}(\omega)=\partial_{\mu} \omega_{\nu}^{a b}+\omega_{\mu}{ }^{a}{ }_{c} \omega_{\nu}{ }^{c b}-(\mu \leftrightarrow \nu)$.

In the definition of operators (15), we replace the ordinary momenta with the covariant ones as well. As a result, the operators cease to obey algebra (8). Therefore, Lagrangian (13) loses the invariance under gauge transformations (10).

To recover the gauge invariance, we do not need to restore total algebra (8), it is enough to ensures the existence of the following commutation relations:

$$
\begin{aligned}
& {\left[L_{1}, L_{-1}\right]=L_{0},} \\
& {\left[L_{2}, L_{-1}\right]=L_{1} .}
\end{aligned}
$$


To restore these relations, let us represent operators (15) as normal ordered functions of the creation and annihilation operators as well as of $R_{\mu \nu \alpha \beta}$ and $R$, i.e.

$$
L_{i}=L_{i}\left(\bar{a}_{\mu}, \bar{b}, a_{\mu}, b, R_{\mu \nu \alpha \beta}, R\right)
$$

The particular form of the operators $L_{i}$ is defined from the condition recovering of commutation relations (22) and (23) by these operators. We should note that it is enough to define the form of the operators $L_{1}$ and $L_{2}$, since the operators $L_{0}$ and $N$ can be expressed in terms of these operators.

Since we have turned to the extended universal enveloping algebra of the Heisenberg algebra, the arbitrariness in the definition of the operators $a$ and $b$ appears. Besides, we should admit the presence of arbitrary operator functions depending on $a, b, R_{\mu \nu}^{a b}$, and $R$ in the right-hand side of (11) and (14). In this, such a modification of the operators must not lead to breaking the Jacobi identity and under the transition to flat space they must restore the initial algebra. However, one can make sure that using the arbitrariness in the definition of the creation and annihilation operators, we can restore algebra (1), (14) at linear order in the Riemann tensor and scalar curvature.

We shall search for the operators $L_{1}$ and $L_{2}$ as series in the Riemann tensor and scalar curvature.

Let us consider linear approximation.

Operator $L_{1}$ should be no higher than linear in the operator $\mathcal{P}_{\mu}$, since the presence of a greater number of these operator changes type of the gauge transformations and the number of physical degrees of freedom. Therefore, in this approximation we can search for them in the form

$$
\begin{aligned}
L_{1}^{(1)}= & R\left(h_{0}(\bar{b}, b) b+h_{1}(\bar{b}, b) b(\bar{a} \cdot a)+\bar{b} h_{2}(\bar{b}, b) a^{2}+h_{3}(\bar{b}, b) b^{3} \bar{a}^{2}\right. \\
& \left.+h_{4}(\bar{b}, b)(\mathcal{P} \cdot a)+h_{5}(\bar{b}, b) b^{2}(\bar{a} \cdot \mathcal{P})\right)+R^{\mu \nu a b}\left(h_{6}(\bar{b}, b) b \bar{a}_{\mu} \bar{a}_{a} a_{\nu} a_{b}\right. \\
& \left.+h_{7}(\bar{b}, b) \bar{a}_{\mu} a_{\nu} \mathcal{P}_{a} a_{b}+h_{8}(\bar{b}, b) b_{2} \bar{a}_{\mu} \mathcal{P}_{\nu} \bar{a}_{a} a_{b}\right)
\end{aligned}
$$

At the same time the operator $L_{2}$ cannot depend on the momentum operators at all, since condition (9) defines the purely algebraic constraint on the coefficient functions. Therefore, at this order we choose the operator $L_{2}$ in the following form:

$$
\begin{aligned}
L_{2}^{(1)}= & R\left(h_{9}(\bar{b}, b) b^{2}+h_{10}(\bar{b}, b) a^{2}+h_{11}(\bar{b}, b) b^{2}(\bar{a} \cdot a)+h_{12}(\bar{b}, b) b^{4} \bar{a}^{2}\right) \\
& +h_{13}(\bar{b}, b) b^{2} \bar{a}^{\mu} \bar{a}^{a} a^{\nu} a^{b} R_{\mu \nu a b} .
\end{aligned}
$$

Here $h_{i}(\bar{b}, b)$ are normal ordered operator functions

$$
h_{i}(\bar{b}, b)=\sum_{n=0}^{\infty} H_{n}^{i} \bar{b}^{n} b^{n},
$$

where $H_{n}^{i}$ are arbitrary real coefficients.

Let us define a particular form of the functions $h_{i}$ from the condition of recovering commutation relations (22) and (23) by the operators $L_{1}$ and $L_{2}$. 
We have to note that these operators can obey relations (22) and (23) up to the terms proportional to $L_{2}^{(0)}=\frac{1}{2}\left(a^{2}+b^{2}\right)$ at linear order, since this does not break the gauge invariance due to constraint (11).

Having calculated (22) and passing to normal symbols of the creation and annihilation operators, we obtain a system of differential equations in the normal symbols of operator functions $h_{i}$. For the normal symbols of operator functions we shall use the same notations. This does not lead to the mess since we consider the operator functions as the ones of two variables, while their normal symbols as functions of one variable. Thereby, we have equations from (22)

$$
\begin{aligned}
& h_{7}^{\prime \prime}(x)+2 h_{7}^{\prime}(x)+4 h_{13}(x)-2 h_{8}(x)=0, \\
& x\left(h_{6}^{\prime \prime}(x)+2 h_{13}^{\prime}(x)+2 h_{6}^{\prime}(x)\right)+2\left(h_{6}^{\prime}(x)+2 h_{13}(x)\right)=0, \\
& x^{2}\left(\frac{1}{2} h_{8}^{\prime \prime}(x)+h_{8}^{\prime}(x)\right)+2 x\left(h_{8}^{\prime}(x)+h_{8}(x)\right)+h_{8}(x)-2 h_{7}(x)=0, \\
& \left(h_{2}^{\prime \prime}(x)+2 h_{12}^{\prime}(x) x+2 h_{2}^{\prime}(x)+8 h_{12}(x)-2 h_{3}(x)\right)=0, \\
& h_{4}^{\prime \prime}(x)+2 h_{4}^{\prime}(x)+2 h_{11}(x)-2 h_{5}(x)=0, \\
& x\left(\frac{1}{2} h_{1}^{\prime \prime}(x)+h_{11}^{\prime}(x)+h_{1}^{\prime}(x)\right)+h_{1}^{\prime}(x)+2 h_{11}(x)+2 h_{2}(x)=0, \\
& x^{2}\left(\frac{1}{2} h_{5}^{\prime \prime}(x)+h_{5}^{\prime}(x)\right)+2 x\left(h_{5}^{\prime}(x)+h_{5}(x)\right)+2 h_{10}(x)+\frac{1}{D} h_{7}(x)+h_{5}(x)=0, \\
& x^{3}\left(\frac{1}{2} h_{3}^{\prime \prime}(x)+h_{3}^{\prime}(x)\right)+3 x^{2}\left(h_{3}^{\prime}(x)+h_{3}(x)\right)+x\left(-\frac{1}{2} h_{0}^{\prime \prime}(x)+h_{10}^{\prime}(x)\right. \\
& \left.-h_{9}^{\prime}(x)-h_{0}^{\prime}(x)+\frac{1}{D} h_{6}(x)+3 h_{3}(x)-h_{2}(x)+h_{1}(x)\right) \\
& -h_{0}^{\prime}(x)-2 h_{9}(x)-D h_{2}(x)=0 .
\end{aligned}
$$

Here the prime denotes the derivative with respect to $x$, while $x=\bar{\beta} \beta$, where $\bar{\beta}$ and $\beta$ are the normal symbols of the operators $\bar{b}$ and $b$, correspondingly.

Similarly, from (23) we derive the other system of equations:

$$
\begin{aligned}
& h_{8}^{\prime}(x)=0, \\
& h_{8}^{\prime \prime}(x) x+h_{7}^{\prime \prime}(x)+3 h_{8}^{\prime}(x)+2 h_{6}^{\prime}(x)=0, \\
& h_{8}^{\prime}(x) x+3 h_{7}^{\prime}(x)+2 h_{8}(x)+2 h_{6}(x)=0, \\
& x^{2} h_{8}^{\prime \prime}(x)+x\left(h_{7}^{\prime \prime}(x)+4 h_{8}^{\prime}(x)+6 h_{6}^{\prime \prime}(x)\right)+h_{7}^{\prime}(x)+2 h_{8}+6 h_{6}-4=0, \\
& h_{6}^{\prime \prime}(x) x+2 h_{6}^{\prime}(x)=0, \\
& h_{8}^{\prime}(x) x+\frac{1}{2} h_{7}^{\prime}(x)+2 h_{8}(x)+h_{6}(x)=0,
\end{aligned}
$$




$$
\begin{aligned}
& x\left(h_{5}^{\prime \prime}(x)+2 h_{3}^{\prime}(x)\right)+h_{4}^{\prime \prime}(x)+3 h_{5}^{\prime}(x)+4 h_{2}^{\prime}(x)+h_{1}^{\prime}(x)+6 h_{3}(x)=0, \\
& h_{1}^{\prime \prime}(x) x+2 h_{1}^{\prime}(x)=0 \\
& h_{5}^{\prime}(x) x+3 h_{4}^{\prime}(x)+2 h_{5}(x)+2 h_{2}(x)+h_{1}(x)=0, \\
& h_{5}^{\prime \prime}(x) x^{2}+\left(h_{4}^{\prime \prime}(x)+4 h_{5}^{\prime}(x)+2 h_{2}^{\prime}(x)+3 h_{1}^{\prime}(x)\right) x+h_{4}^{\prime}(x)+2 h_{5}(x) \\
& +2 h_{2}(x)+3 h_{1}(x)=0 \\
& h_{3}^{\prime \prime}(x) x^{2}+\left(h_{2}^{\prime \prime}(x)+6 h_{3}^{\prime}(x)\right) x+2 h_{2}^{\prime}(x)+6 h_{3}(x)=0 \\
& h_{3}^{\prime \prime}(x) x^{3}+\left(h_{2}^{\prime \prime}(x)+4 h_{3}^{\prime}(x)\right) x^{2}-2 h_{0}^{\prime \prime}(x) x-4 h_{0}^{\prime}(x)=0 .
\end{aligned}
$$

Having solved the whole system of equations (26) and (27), we obtain the particular form of the operators $L_{1}$ and $L_{2}$ :

$$
\begin{aligned}
L_{1}^{(1)}= & \frac{1}{6} R^{\mu \nu \alpha \beta} \bar{\alpha}_{\alpha} \alpha_{\mu}\left\{\mathcal{P}_{\nu} \alpha_{\beta}(1+2 \bar{\beta} \beta)-5 \bar{\alpha}_{\nu} \alpha_{\beta} \beta+2 \bar{\alpha}_{\nu} \mathcal{P}_{\beta} \beta^{2}\right\} \\
& +R\left\{c_{2}(\mathcal{P} \cdot \alpha)+c_{1} \beta-\frac{1}{2} \alpha^{2} \bar{\beta}^{2} h_{5}^{\prime}(x) \beta-\alpha^{2} \bar{\beta} h_{5}(x)\right. \\
& \left.+(\bar{\alpha} \cdot \mathcal{P}) h_{5}(x) \beta^{2}+\frac{1}{2} \bar{\alpha}^{2} h_{5}^{\prime}(x) \beta^{3}\right\}, \\
L_{2}^{(1)}= & R\left\{\alpha ^ { 2 } \left(-\frac{1}{4} \bar{\beta}^{2} h_{5}^{\prime \prime}(x) \beta^{2}-\frac{1}{2} \bar{\beta}^{2} h_{5}^{\prime}(x) \beta^{2}-\bar{\beta} h_{5}^{\prime}(x) \beta-\bar{\beta} h_{5}(x) \beta\right.\right. \\
& \left.-\frac{1}{2} h_{5}(x)+\frac{1}{6 D} \bar{\beta} \beta+\frac{1}{12 D}\right)+\frac{1}{4} \bar{\alpha}^{2}\left(h_{5}^{\prime \prime}(x)+2 h_{5}^{\prime}(x)\right) \beta^{4} \\
& \left.+(\bar{\alpha} \cdot \alpha) h_{5} \beta^{2}+\frac{1}{2} h_{5}(x) \beta^{2} D+\frac{1}{3 D} \bar{\beta} \beta^{3}\right\},
\end{aligned}
$$

where $c_{1}$ and $c_{2}$ are arbitrary real parameters and $h_{5}(x)$ is an arbitrary function regular at $x \rightarrow 0$, while $\bar{\alpha}_{\mu}$ and $\alpha_{\mu}$ are normal symbols of the operators $\bar{a}_{\mu}$ and $a_{\mu}$. One can verify that the function $h_{5}(x)$ corresponds to the rest of the arbitrariness in the redefinition of the creation and annihilation operators when initial Heisenberg algebra (1), (14) is fixed. Therefore, we can set $h_{5}(x)=0$.

The transition to the operator functions is realized in the conventional manner:

$$
: O(\bar{a}, \bar{b}, a, b):=\left.\exp \left(\bar{a} \cdot \frac{\partial}{\partial \bar{\alpha}}\right) \exp \left(\bar{b} \frac{\partial}{\partial \bar{\beta}}\right) \exp \left(a \cdot \frac{\partial}{\partial \alpha}\right) \exp \left(b \frac{\partial}{\partial \beta}\right) O(\bar{\alpha}, \bar{\beta}, \alpha, \beta)\right|_{\substack{\alpha \# \rightarrow 0 \\ \beta \# \rightarrow 0}} .
$$

Thus, we have obtained the general form of the operators $L_{n}$, which satisfy commutation relations (22) and (23) in linear approximation. This means that Lagrangian (13) is invariant under gauge transformations (10) at this order. The form of the operator $L_{2}$ has changed in this approximation, hence, the conditions

$$
L_{2} L_{2}\left|\Phi^{s}\right\rangle=0, \quad L_{2}\left|\Lambda^{s-1}\right\rangle=0
$$

undergo the nontrivial modifications in terms of the coefficient functions.

\footnotetext{
${ }^{5}$ We search for regular at $x \rightarrow 0$ solutions only.
} 


\section{Examples}

In this section we apply the proposed algebraic scheme to the description of propagation of the massive states with spin 1 and 2 in the Symmetrical Einstein space.

Vector massive field. This case is quite interesting since it is practically the only massive bosonic field among the other higher-spin states which was observed in the experiment. Let us consider the state in the Hilbert space that corresponds to the massive state with spin 1.

$$
|1\rangle=((v \cdot \bar{a})+\varphi \bar{b})|0\rangle
$$

It is not difficult to compute the expectation value of operator (13) in this state. Having calculate this, one derive, the following Lagrangian[ in linear approximation

$$
\begin{aligned}
\mathcal{L}_{s=1}= & \left(1+2 c_{2} R\right)\left(\bar{v}^{\alpha} \mathcal{P}^{2} v_{\alpha}-\bar{v}^{\beta} \mathcal{P}_{\beta} \mathcal{P}_{\alpha} v^{\alpha}+\bar{\varphi} \mathcal{P}^{2} \varphi\right)+\left(1+2 c_{1} R\right) \bar{v}^{\alpha} v_{\alpha} \\
& -\left(1+\left(c_{1}+c_{2}\right) R\right)\left(\bar{\varphi} \mathcal{P}_{\alpha} v^{\alpha}+\text { h.c. }\right)+\frac{1}{3} \bar{v}_{\delta} R^{\alpha \delta \gamma \beta} \mathcal{P}_{\alpha} \mathcal{P}_{\gamma} v_{\beta} .
\end{aligned}
$$

For the Lagrangian to describe the massive vector field properly, we have to impose the constraints

$$
1+2 c_{1} R \geq 0, \quad 1+2 c_{2} R>0 .
$$

The former constraint ensures the given state not to be the tachyon, while the latter one provides kinetic terms with the right sign.

The gauge vector for the massive spin-1 state is

$$
|\Lambda, 1\rangle=\eta b|0\rangle
$$

and the gauge transformations for the massive field are

$$
\begin{aligned}
\delta v_{\alpha} & =\left(1+c_{2} R\right) \mathcal{P}_{\alpha} \eta \\
\delta \varphi & =\left(1+c_{1} R\right) \eta
\end{aligned}
$$

For the vector massive field it is not difficult to generalize the linear approximation to the general case] of arbitrary symmetrical Einstein space. For that we make the following substitution:

$$
c_{1} R \rightarrow f_{1}(R), \quad c_{2} R \rightarrow f_{2}(R) .
$$

But the gauge invariance requires the functions to be equal to each other. Thereby, the whole Lagrangian describing the propagation of the vector field in the considered background is

$$
\begin{aligned}
\mathcal{L}_{s=1}= & (1+f(R))\left(\bar{v}^{\alpha} \mathcal{P}^{2} v_{\alpha}-\bar{v}^{\beta} \mathcal{P}_{\beta} \mathcal{P}_{\alpha} v^{\alpha}+\bar{\varphi} \mathcal{P}^{2} \varphi+\bar{v}^{\alpha} v_{\alpha}-\left(\bar{\varphi} \mathcal{P}_{\alpha} v^{\alpha}+\text { h.c. }\right)\right) \\
& +\frac{1}{3} \bar{v}_{\delta} R^{\alpha \delta \gamma \beta} \mathcal{P}_{\alpha} \mathcal{P}_{\gamma} v_{\beta} .
\end{aligned}
$$

There is no reason to be surprised, since, due to the gauge invariance, we cannot obtain a different result by virtue of the fact that $R$ is constant.

\footnotetext{
${ }^{6}$ We suppress the usual factor $\sqrt{-g}$.

${ }^{7}$ Of course, this is only one possibility among others.
} 
Now we can consider two variants. The first is when $1+f(R)>0$. Then, the Lagrangian is invariant under the usual gauge transformations for the massive vector fields

$$
\begin{aligned}
\delta v_{\mu} & =\mathcal{P}_{\mu} \eta, \\
\delta \varphi & =\eta .
\end{aligned}
$$

In principle, in this case we can include the multiplier $1+f(R)$ into the normalization of fields. After that we obtain the usual Lagrangian "minimally" coupled to the Riemann background with the single "non-minimal" term.

A different quite unusual situation is realized when $1+f(R)=0$. Then, the whole Lagrangian consists of the single term

$$
\mathcal{L}=\frac{1}{3} R^{\mu \nu \alpha \beta} \bar{V}_{\mu \nu} V_{\alpha \beta},
$$

where $V_{\mu \nu}=\partial_{\mu} v_{\nu}-\partial_{\nu} v_{\mu}$. Obviously, the Lagrangian is invariant under the transformation $\delta v_{\mu}=\mathcal{P}_{\mu} \eta$. One can notice that the transition to an arbitrary Riemann space does not break this invariance.

Let us discuss the causality for the massive vector field in the given background. Having fixed the gauge invariance by

$$
\varphi=0
$$

from (29) we derive the following equations

$$
\frac{\delta \mathcal{L}}{\delta \bar{v}^{\mu}}=\left(1+2 c_{2} R\right)\left(\mathcal{P}^{2} v_{\mu}-\mathcal{P}_{\mu}(\mathcal{P} \cdot v)\right)+\left(1+2 c_{1} R\right) v_{\mu}-\frac{1}{3} R_{\mu}{ }^{\nu \alpha \beta} \mathcal{P}_{\nu} \mathcal{P}_{\alpha} v_{\beta}
$$

Having taken the divergence of these equations one obtains the constraint

$$
\mathcal{P}^{\mu} \frac{\delta \mathcal{L}}{\delta \bar{v}^{\mu}}=\left(1+\left(2\left(c_{1}-c_{2}\right)-\frac{1}{D}\right) R\right)(\mathcal{P} \cdot v)+\mathcal{O}\left(R^{2}\right)=0 .
$$

From here we see that if we impose the requirement

$$
m^{2}+\left(2\left(c_{1}-c_{2}\right)-\frac{1}{D}\right) R \neq 0
$$

we get the necessary constraint on the vector field at this order. Here we have restored the dimensional parameter $m$. Calculating the characteristic determinant $\left.\right|^{\text {f }}$, we obtain as a result

$$
D(n)=\left(3 D m^{2}+R\right)\left(n^{2}\right)^{D}+\mathcal{O}\left(R^{2}\right)
$$

where $n_{\mu}$ is the normal vector to the characteristic surface. The equations of motion will be causal (hyperbolic) if the solutions $n^{0}$ to $D(n)=0$ are real for any $\vec{n}$. Thereby, in our case, from the condition $D(n)=0$ we have the usual light cone as the solution for $n_{\mu}$ if we impose the following condition:

$$
3 D m^{2}+R \neq 0
$$

Of course, our consideration essentially depends on the higher orders in the Riemann tensor and scalar curvature.

Thus, we can see that in such theory there are restrictions on $m$ and $R$ similar to Ref. [23, 24.

${ }^{8}$ The massive vector field becomes a ghost when $1+f(R)<0$.

${ }^{9}$ The determinant is entirely determined by the coefficients at the highest derivatives in equations of motion after gauge fixing and resolving all the constaraints [22]. 
Massive field with spin 2. Now we obtain the Lagrangian describing the propagation of massive spin-2 field in the symmetrical Einstein space. The following state in the Fock space corresponds to such field

$$
|2\rangle=\left\{(\bar{a} \cdot h \cdot \bar{a})+(v \cdot \bar{a}) \bar{b}+\varphi b^{2}\right\}|0\rangle .
$$

It is easy to see that this state trivially satisfies condition (10).

Having calculated the expectation value of operator (13) in this state we derive the following Lagrangian

$$
\begin{aligned}
\mathcal{L}_{s=2}= & \left(1+2 c_{2} R\right)\left\{\bar{h}^{\alpha \beta} \mathcal{P}^{2} h_{\alpha \beta}-2 \bar{h} \mathcal{P}^{2} h-2 \bar{h}^{\beta \gamma} \mathcal{P}_{\beta} \mathcal{P}_{\alpha} h_{\gamma}^{\alpha}+\left\{2 \bar{h} \mathcal{P}_{\alpha} \mathcal{P}_{\beta} h^{\alpha \beta}\right.\right. \\
& \left.\left.+\bar{\varphi} \mathcal{P}_{\alpha} \mathcal{P}_{\beta} h^{\alpha \beta}-\bar{h} \mathcal{P}^{2} \varphi+h . c .\right\}+\frac{1}{2} \bar{v}^{\alpha} \mathcal{P}^{2} v_{\alpha}-\frac{1}{2} \bar{v}^{\beta} \mathcal{P}_{\alpha} \mathcal{P}_{\beta} v^{\alpha}\right\} \\
& -\left(1+\left(c_{1}+c_{2}\right) R\right)\left\{\bar{v}_{\beta} \mathcal{P}_{\alpha} h^{\alpha \beta}-\bar{h} \mathcal{P}_{\alpha} v^{\alpha}+h . c .\right\} \\
& +\left(1+2 c_{1} R\right)\left(\bar{h}^{\alpha \beta} h_{\alpha \beta}-\bar{h} h\right)-\frac{3}{8} R^{\alpha \gamma \beta \delta} \bar{h}_{\gamma \mu} \mathcal{P}_{\alpha} \mathcal{P}_{\delta} h_{\beta}^{\mu} \\
& -\frac{1}{2} R^{\alpha \gamma \beta \delta} \bar{v}_{\gamma} \mathcal{P}_{\alpha} \mathcal{P}_{\delta} v_{\beta}-\frac{1}{6}\left\{2 R^{\alpha \gamma \beta \delta} \bar{h}_{\beta \gamma} \mathcal{P}_{\alpha} \mathcal{P}_{\mu} h_{\delta}^{\mu}-R^{\alpha \gamma \beta \delta} \bar{h}_{\beta \gamma} \mathcal{P}_{\alpha} \mathcal{P}_{\delta} h\right. \\
& \left.-5 R^{\alpha \gamma \beta \delta} \bar{h}_{\beta \gamma} \mathcal{P}_{\alpha} \mathcal{P}_{\delta} \varphi+5 R^{\alpha \gamma \beta \delta} \bar{h}_{\beta \gamma} \mathcal{P}_{\alpha} v_{\delta}+h . c .\right\}+\frac{4}{3} R^{\alpha \gamma \beta \delta} \bar{h}_{\alpha \beta} h_{\gamma \delta} \\
& +\frac{1}{3 D} R\left\{-\bar{h} \mathcal{P}^{2} h+\left\{\frac{1}{2} \bar{h} \mathcal{P}_{\alpha} \mathcal{P}_{\beta} h^{\alpha \beta}-\frac{1}{2} \bar{h} \mathcal{P}^{2} \varphi+h . c .\right\}+3 \bar{\varphi} \mathcal{P}^{2} \varphi\right. \\
& \left.+\left\{\frac{1}{2} \bar{v}^{\alpha} \mathcal{P}_{\alpha} h-3 \bar{\varphi} \mathcal{P}_{\alpha} v^{\alpha}+h . c .\right\}-4 \bar{h} h+3 \bar{v}^{\alpha} v_{\alpha}\right\}
\end{aligned}
$$

where $h=g^{\mu \nu} h_{\mu \nu}$. It is not difficult to notice that we have to impose the same restrictions (30) for the proper description of the massive state.

The gauge vector for the massive spin-2 state is

$$
|\Lambda, 2\rangle=\{(\xi \cdot \bar{a})+\eta b\}|0\rangle .
$$

Condition (11) is the non-trivial constraint for the gauge vectors of massive states with spin 3 and higher only.

From (10) we obtain the following gauge transformations

$$
\begin{aligned}
\delta h_{\alpha \beta} & \left.=\left(1+c_{2} R\right) \mathcal{P}_{(\alpha} \xi_{\beta)}-\frac{1}{6} R_{(\alpha}{ }^{\gamma} \beta\right) \\
\delta & \mathcal{P}_{\gamma} \xi_{\delta}, \\
\delta v_{\alpha} & =\left(1+c_{2} R\right) \mathcal{P}_{\alpha} u+\left(1+c_{1} R\right) \xi_{\alpha}, \\
\delta \varphi & =\left(1+c_{1} R\right) \eta .
\end{aligned}
$$

\footnotetext{
${ }^{10}$ One can verify that condition (9) imposes a not-trivial restriction only on the states with spin 4 and higher.
} 
Let us fix the gauge invariance by means of the gauge condition

$$
v_{\mu}=0, \quad \varphi=0 .
$$

Now we have the following equations of motion

$$
\begin{aligned}
\frac{\delta \mathcal{L}}{\delta h^{\mu \nu}}= & \left(1+2 c_{2} R\right)\left(\mathcal{P}^{2} h_{\mu \nu}-2 \mathcal{P}_{(\mu}(\mathcal{P} \cdot h)_{\nu)}+\mathcal{P}_{(\nu} \mathcal{P}_{\mu)} h\right. \\
& \left.-g_{\mu \nu}\left(\mathcal{P}^{2} h-(\mathcal{P} \cdot \mathcal{P} \cdot h)\right)\right)-\left(1+2 c_{1} R\right)\left(h_{\mu \nu}-g_{\mu \nu} h\right) \\
& +\frac{R}{6 D}\left(\mathcal{P}_{(\nu} \mathcal{P}_{\mu)} h-g_{\mu \nu}\left\{2 \mathcal{P}^{2} h-(\mathcal{P} \cdot \mathcal{P} \cdot h)-8 h\right\}\right) \\
& -\frac{1}{6} g_{\mu \nu} R^{\alpha \delta \beta \gamma} \mathcal{P}_{\delta} \mathcal{P}_{\gamma} h_{\alpha \beta}+\frac{1}{3} R_{\alpha(\mu \mid \beta}{ }^{\gamma} \mathcal{P}_{\mid \nu)} \mathcal{P}_{\gamma} h^{\alpha \beta}-\frac{1}{6} R^{\beta}{ }_{(\mu}^{\gamma}{ }_{\nu} \mathcal{P}_{\gamma} \mathcal{P}_{\beta} h \\
& +\frac{1}{3} R_{\beta\left(\mu^{\gamma}{ }_{\nu}\right)} \mathcal{P}_{\gamma}(\mathcal{P} \cdot h)^{\beta}-\frac{2}{3} R^{\alpha \gamma \beta}{ }_{(\mu \mid} \mathcal{P}_{\gamma} \mathcal{P}_{\beta} h_{\mid \nu) \alpha}-\frac{4}{3} R_{\alpha \mu \beta \nu} h^{\alpha \beta}
\end{aligned}
$$

From these equations we can obtain the constraints:

$$
\begin{aligned}
& (\mathcal{P} \cdot h)_{\mu}=\mathcal{P}_{\mu} h\left(1+\frac{R}{6 D}\right)+\frac{5}{6} R_{\mu}{ }^{\nu \alpha \beta} \mathcal{P}_{\alpha} h_{\nu \beta}, \\
& \left(3+2\left(3 c_{1} D+\frac{2}{D}\right) R\right) h=0 .
\end{aligned}
$$

Now one can see that when

$$
3+2\left(3 c_{1} D+\frac{2}{D}\right) R \neq 0
$$

we have the appropriate number of the constraints and, correspondingly, the appropriate number of physical degree of freedom at this order.

Now we consider the question of causality of the massive spin-2 state in linear approximation. Using relations (36) and the equations of motion (35), one can obtain the characteristic determinant

$$
D(n)=\left(n^{2}\right)^{\frac{D^{2}(D+1)^{2}}{4}}\left(1+\frac{D+5}{3 D} R\right)+\mathcal{O}\left(R^{2}\right) .
$$

Thereby, when $\left(1+\frac{D+5}{3 D} R\right) \neq 0$ from the condition $D(n)=0$, we have the usual light cone in this approximation, i.e. the causal propagation of the massive spin-2 state in the considered Riemann background.

For the massive states with higher spins, one can derive a similar result. Quite obviously that the causal propagation of these states in considered background imposes some restrictions on the mass of states and the scalar curvature only.

\section{Conclusion}

We have applied the algebraic scheme proposed in Ref. [15, 16] to the description of propagation of the gauge massive fields in the arbitrary symmetrical Einstein space of arbitrary dimensionality in the lowest approximation in the Riemann tensor and scalar curvature. 
This approach is quite convenient since it allows one to reduce the cumbersome problem of searching for the gauge invariant action of higher-spin fields to the pure algebraic problem of search for the appropriate modification of some operators. In principal, this approach can be applied to the description of fermionic massive fields in such background as well.

\section{Acknowledgment}

The author is grateful to $\mathrm{Yu}$. M. Zinoviev for the numerous useful discussions and the help in the work.

\section{References}

[1] C. Aragone and S. Deser, Phys. Lett., 86B (1979), 161.

[2] M. A. Vasilev, Yad. Fiz. 45 (1987) 1784; ibid. 47 (1988) 831; ibid. 48 (1988) 1478.

[3] M. A. Vasilev and E. S. Fradkin, Jetp Lett. 44 (1986) 484 (in russian); Phys. Lett. B189 (1987) 89; Nucl. Phys. B291 (1987) 141.

[4] M. A. Vasilev, Int. J. Mod. Phys., D5 (1996), 763, hep-th/9611024.

[5] James T. Liu Ioannis Giannakis and Massimo Porrati, Massive higher spin states in string theory and the principle of equivalence. Preprint CERN-TH-98-304, hepth/9809142.

[6] Massimo Porrati, Phys. Lett., B304 (1993), 77-80, gr-qc/9301012.

[7] A. Cucchieri, M. Porrati, and S. Deser, Phys. Rev., D51 (1995), 4543-4549, hepth/9408073.

[8] L. P. C. Singh and C.R. Hagen, Phys. Rev, D9 (910), 898, 1974.

[9] Yu. M. Zinoviev, Gauge invariant description of massive high spin particles. IHEP preprint 83-91 (Protvino, 1983).

[10] Yu. M. Zinoviev, The Role of Poincare Group in Elementary PArticle Physics, XVII Seminar on High Energy Physics and Field Theory, June 1994, Protvino.

[11] S. M. Klishevich and Yu. M. Zinoviev, Phys. Atom. Nucl., 61 (1998), 1527-1537, hepth/9708150.

[12] A.I. Pashnev, Teor. Mat. Fiz., 78, 1989 (in Russian).

[13] P. C. Argyres and C. R. Nappi, Phys. Lett., B224 (1989), 89.

[14] J. Fang and C. Fronsdal, J. of Mat. Phys (N.Y.), 20 (1979), 2264.

[15] S. M. Klishevich, Massive fields with arbitrary integer spin in homogeneous electromagnetic field. 1998, Preprint IHEP 98-24, hep-th/9810228. 
[16] S. M. Klishevich, Massive fields with arbitrary half-integer spin in constant electromagnetic field. 1998, Preprint IHEP 98-70, hep-th/9811030.

[17] J. M. F. Labastida, Phys. Rev. Lett. 58 (1987) 531; Nucl. Phys. B322 (1989) 185.

[18] A. Pashnev and M. Tsulaya, Mod. Phys. Lett., A12 (1997), 861-870, hep-th/9703010.

[19] C. Fronsdal, Phys. Rev., D18 (1978), 3624.

[20] T. Curtright, Phys. Lett, 85B (1979), 219.

[21] A. Barducci and D. Dominici, Nuovo Cim., 37A (1977), 385.

[22] G. Velo and D. Zwanziger, Phys. Rev., 186 (1969) 1337.

[23] Atsushi Higuchi, Nucl. Phys., B282 (1987), 397.

[24] I. Bengtsson, J. Math. Phys., 36 (1995), 5805, gr-qc/9411057. 